\title{
Design Strategy for a New High-G Accelerometer
}

\author{
Robert Kuells ${ }^{1}$, Siegfried Nau ${ }^{1}$, Christian Bohland ${ }^{1}$, Manfred Salk ${ }^{1}, K^{1}$ Klaus Thoma ${ }^{1}$, \\ ${ }^{1}$ Fraunhofer Institute for High-Speed Dynamics, Ernst-Mach-Institut (EMI), \\ Am Klingelberg 1, 79588 Efringen-Kirchen, Germany \\ robert.kuells@emi.fraunhofer.de
}

\begin{abstract}
:
A new piezoresistive high-g accelerometer is presented that was developed on the basis of an unconventional, displacement focused design approach. The approach analyses the "geometrical constant", which describes the relation between the square of the resonant frequency and the displacement under static load of a spring-mass system. The value is maximized in order to achieve a high sensitivity, high bandwidth accelerometer. It has a figure of merit (sensitivity times resonant frequency squared) of about $5 \cdot 10^{6} / \mathrm{m}$, which is higher than any other accelerometer in the literature or on the market. The design has been implemented as a MEMS device and has been characterized for shock loads up to $60,000 \mathrm{~g}$. The concept of the geometrical constant may be applicable to other sensor types.
\end{abstract}

Key words: MEMS design, geometrical constant, accelerometer, figure of merit, high-g

\section{Introduction}

High-g accelerometers are designed to work in harsh environments, withstanding and measuring extreme mechanical loading. The measurement range addressed by the presented sensor is up to $100,000 \mathrm{~g}$, which forms the high end of useful and technically feasible MEMS accelerometry. Applications are, for example, the study of penetration processes [1], shock testing of electronics [2] and dynamic material characterization [3].

The acceleration pulses that occur in these environments usually are of high amplitude and short duration (micro- to milliseconds) and carry high frequency content. Therefore, the utilized accelerometers need to have very high bandwidth. Additionally, the sensors are also supposed to have good sensitivity, to increase the resolution of the measurement signal.

Concerning bandwidth, accelerometers are limited by the fact that they employ a mechanical spring-mass system to convert acceleration into a measureable displacement. The useful bandwidth then is a fraction of the mechanical resonant frequency of the springmass system. It is well known, that a demand for high sensitivity is opposing that for high bandwidth, which is due to the fact that the two are interrelated, as will be explained in more detail in section 3. Mathematically, this can be expressed by the following equation [4] [5]:

$$
S \omega^{2}=\phi_{\text {design }}
$$

Here, $\omega$ is the first resonant frequency of the device and $S$ its sensitivity. The product $\phi_{\text {design }}$ is a design specific constant of the sensor, illustrating the impossibility of optimizing sensitivity and bandwidth independently. The maximum of the product is limited by the value of this constant, making it a figure of merit. It allows the comparison of accelerometer designs from different sensitivity and bandwidth ranges.

The new accelerometer is, as most high-g MEMS accelerometers are, a piezoresistive inertial device. First experimental results have been published [6] together with a description of the design and design process. Besides showing new experimental results, this paper will focus on discussing the so called "geometrical constant", which is a fundamental property of any spring-mass system. It forms the basis of the design strategy used for accelerometer geometry development and may be generalized to cover other sensor principles.

\section{Motivation of New Design Strategy}

The design of accelerometers is usually discussed on two levels. On the higher one, i.e. the macro level, general features like the sensor type, the general shape of the springmass system or the readout type are qualitatively defined, often depending on previous knowledge. On the lower level, i.e. micro level, a detailed, quantitative optimization of a specific initial geometry is conducted with 
the help of analytical expressions and numerical simulations, arriving at an improved design.

It is the authors' impression that the initial designs, especially their geometrical shape, are either based on previous concepts or justified by their final performance. Both approaches are perfectly sound, but are missing the additional insight a strategy on an intermediate level may provide and seldom discuss how the fundamental geometry was found in the first place. Some research has been conducted in the area of generic topology optimization (see for example [7]). However, no experimental data has been presented.

The new accelerometer design strategy includes an intermediate level, where semiquantitative arguments are established. This meso level is displacement focused and gives the designer a general guideline, helping to quickly identify promising initial geometries. Figure 1 gives a simplified view of the design steps taken on the different working levels. The discussion in this paper will focus on the "geometrical constant". It is a general property of continuous spring-mass systems and therefore is applicable to other sensor types, as well (see section 8).

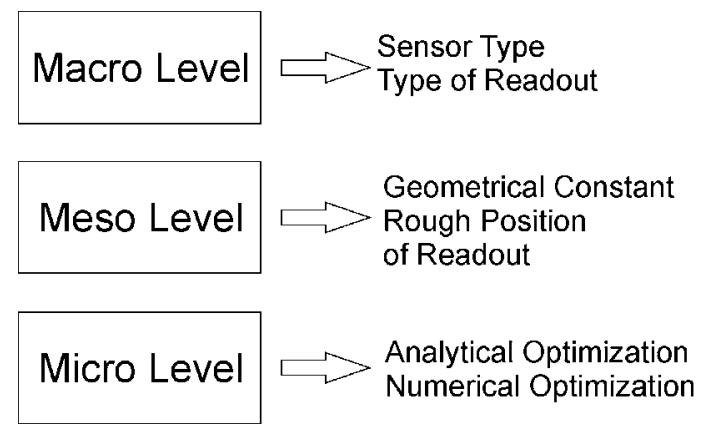

Fig. 1: Working steps at different accelerometer design levels. Manufacturing limitations always have to be kept in mind.

\section{Geometrical Constant}

As mentioned in the introduction, the springmass system is the limiting factor for the bandwidth of an accelerometer. Also, it greatly influences its final sensitivity $S$, since $S$ is proportional (or at least monotonically related) to the amount of displacement $\Delta x$ of the mass for a given acceleration $a$. It is well known, that the spring-mass system is also responsible for the relationship between sensitivity and bandwidth shown in eq. (1). For a single degree of freedom system it holds true that:

$$
\frac{\Delta x}{a} \omega^{2}=1
$$

This formula is quoted when the general sensitivity-bandwidth limitation of accelerometers is discussed (see for example [5] or [8]). However, this formula is inaccurate, if not to say wrong, if applied in general. It is only true for single degree of freedom systems. In a real, continuous system the correct relation is:

$$
\frac{\Delta x_{\max }}{a} \omega^{2}=C
$$

Here, $\Delta x_{\max }$ is the maximum value of the system's deflection curve $\Delta x, \omega$ the eigenfrequency of the corresponding oscillation mode (usually lowest) and $C$ is a dimensionless quantity, i.e. the aforementioned geometrical constant. $C$ depends solely on the relative geometry of the spring-mass system and typically has a value of ' 1 ' to ' 3 '.

Eq. (3) itself is in principle known [9] [10] [11], however, to the authors' knowledge, has never been applied to sensor design. Considering the regular quotation of eq. (2), the geometrical constant almost seems like a forgotten parameter. Yet, its value determines the maximum sensitivity-bandwidth product a certain spring-mass system has and is well worth to be analyzed.

Sundararajan [10] gives an integral to approximate $C$ for a general load. It can be simplified for the case of inertial forces acting on the system:

$$
C \approx \frac{\int_{V} \rho \cdot \Delta x_{\text {norm }} d V}{\int_{V} \rho \cdot \Delta x_{\text {norm }}^{2} d V}
$$

With:

$$
\Delta x_{n o r m}=\frac{\Delta x}{\Delta x_{\max }} \leq 1
$$

Here, $\rho$ is the density of the spring-mass systems material and $\Delta x_{n o r m}$ is the normalized deflection curve of the system. Both quantities can be spatially dependent and are integrated over the physical domain $V$ of the system (in real systems a volume).

The result of the integral is close to ' 1 ', if most of the mass of the spring-mass system is close to its point of maximum deflection. If the mass is distributed in regions of small displacement, the value of $C$ becomes larger. This general behavior is a good guideline for the designer to find starting geometries for subsequent detailed treatment. Figure 2 shows several 2D examples, visualizing the concept. Note that the "classic" geometry with a large mass at the end 
of a bending element has the smallest value for C.

In practice, the integral is not useful to calculate specific values of the geometrical constant, since the deflection curves for anything but simple systems are not readily available. It is more feasible to calculate $C$ with numerical methods, e.g. FEM simulations, as has been done for the examples in figure 2. Numerous further examples can be found in [11].
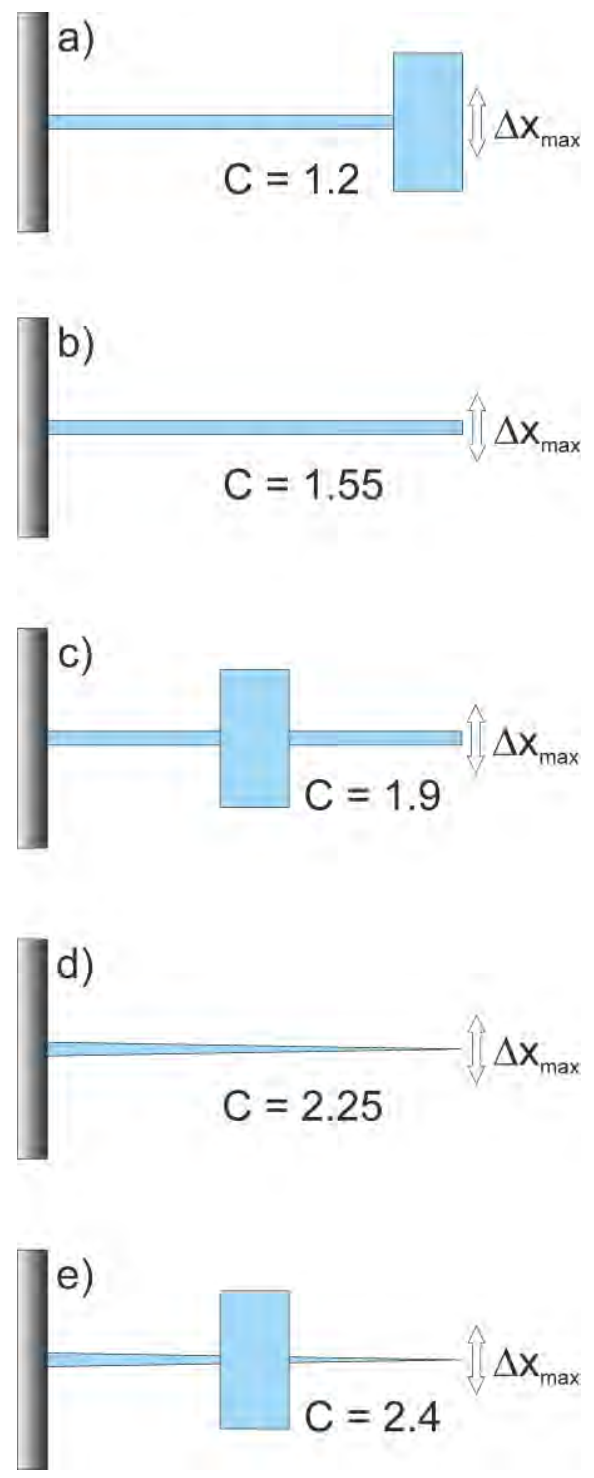

Fig. 2: Geometrical constant of different cantilever configurations. The material density is homogenous in all examples. (Image: [6])

The fact that $C$ is dimensionless is helpful for designing optimized geometries, since it means a spring-mass system can be scaled in size without changing the geometrical constant. The designer can therefore choose an optimized spring-mass system and then scale it in size to have the desired sensitivity-bandwidth tradeoff (within the limitations of the manufacturing process, of course). The mechanical resonant frequency of a spring-mass system is simply anti-proportional to the spatial scaling factor.

The concept of the geometrical constant forms the basis of the new displacement focused design approach. $C$ is to be maximized to obtain maximum deflection per acceleration of the mass for a given resonant frequency. The next step is to efficiently detect this displacement.

\section{Choice of Spring-Mass System}

As mentioned in the previous section, the geometry of the spring-mass system has to be compliant with the limitations of available MEMS processes. For the high-g accelerometer, a plate with constant thickness was chosen, resulting in a geometrical constant of about '2' (see figure 3).

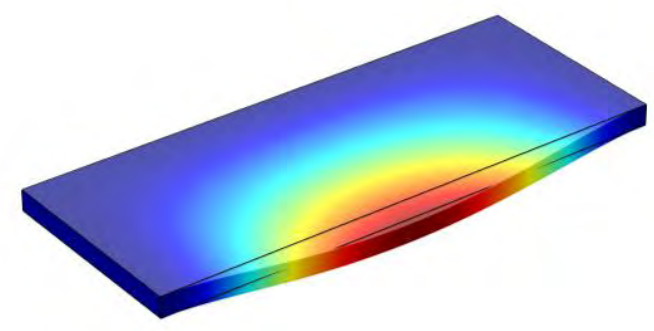

Fig. 3: Spring-mass system of the new accelerometer. It is a flexural plate with three fixed edges. The coloring indicates the displacement curve under load (red: maximum, displacement not drawn to scale).

\section{Further Design Choices}

A piezoresistive readout mechanism is chosen to detect the deflection of the flexural plate. It is simple to manufacture and can be easily connected in a Wheatstone bridge. Singlecrystal silicon is selected as the main material of the sensor. It can be machined with standard processes and allows the creation of piezoresistive elements with very high gauge factors.

Figure 4 shows a schematic of the design with the flexural plate being defined by two trenches that are etched into bulk silicon. The piezoresistive elements, i.e. strain gauges, are free-standing bridges that span these trenches and are connected to the plate and the frame. They are attached close to the center of the plate, in order to maximize the sensitivity of the device. More details about the device design, including a finite element simulation, can be found in [6]. 


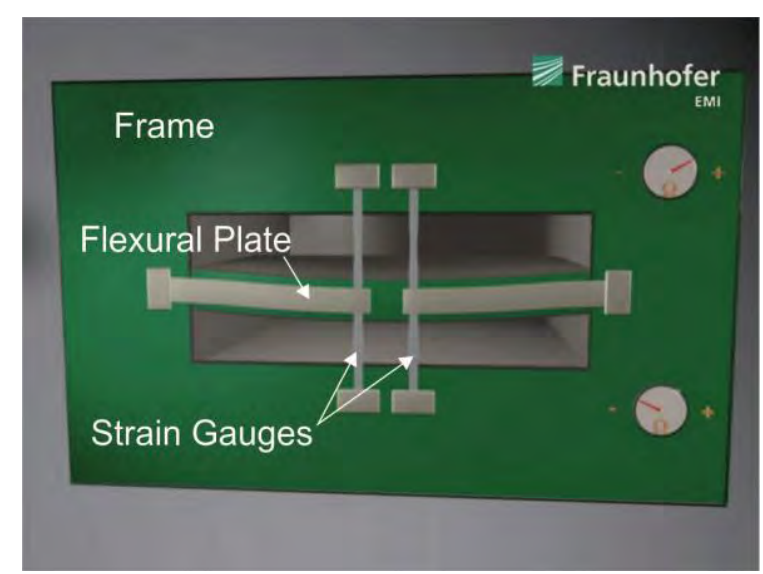

Fig. 4: Schematic of the new design. Free standing piezoresistive bridges span the trenches that define the flexural plate to be the spring-mass element. (Image: [6])

\section{Manufactured Device}

An image of the bare silicon die of the new MEMS accelerometer is shown in figure 5 (top). The silicon processing was conducted together with the Fraunhofer EMFT. A multi-layer LTCC package has been designed together with the Fraunhofer IKTS. Figure 5 (bottom) shows the package, which is SMD mountable.

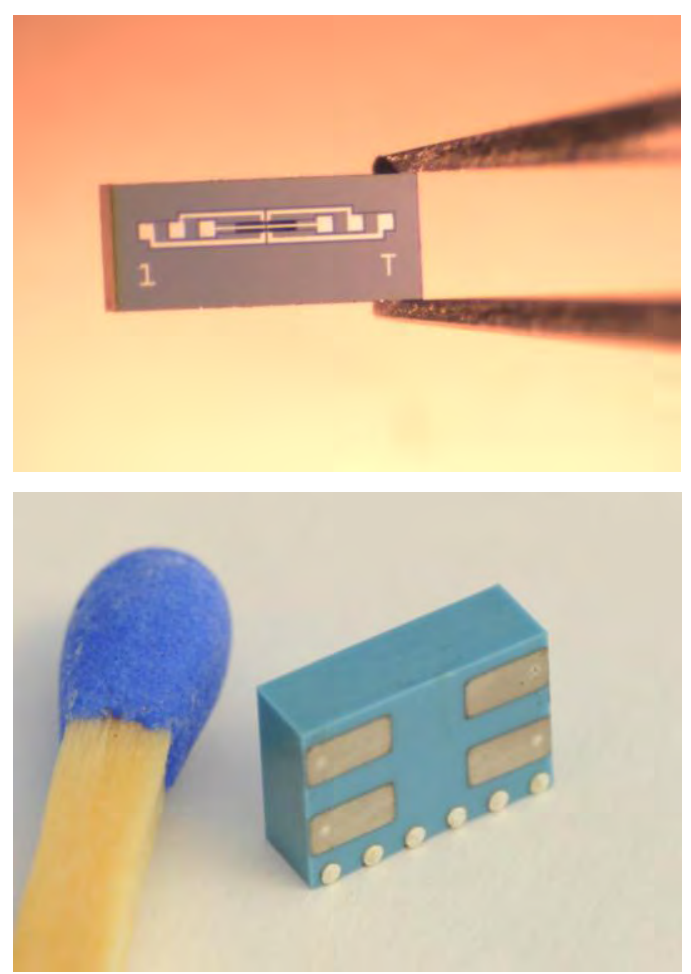

Fig. 5: Above: Image of the bare silicon die of the new design. Below: The packaged sensor with SMD capable metallization.
Note that the new geometry has its sensitive axis in the wafer plane, which is unusual for high-g accelerometers. In [12] it is discussed how this influences the package design, since the requirements of good mechanical coupling and easy electrical contacting both have to be taken into account.

\section{Experimental Results}

The new devices are characterized on a so called Hopkinson bar. This apparatus is capable of reproducibly generating short acceleration shocks with amplitudes of several ten thousand g's. Details on the device and its working principle can for example be found in [13]. Figure 6 (top) shows a sample measurement with a $60,000 \mathrm{~g}$ pulse that is well reproduced by the sensor. It shows the typical half-sine shaped acceleration pulse of the bar together with inevitable residual oscillations of the experimental sensor carrier, which are also measured by the sensor. Figure 6 (bottom) shows a series of three Hopkinson bar tests, which demonstrates the good reproducibility of the loading conditions and the measurement quality of the sensor.
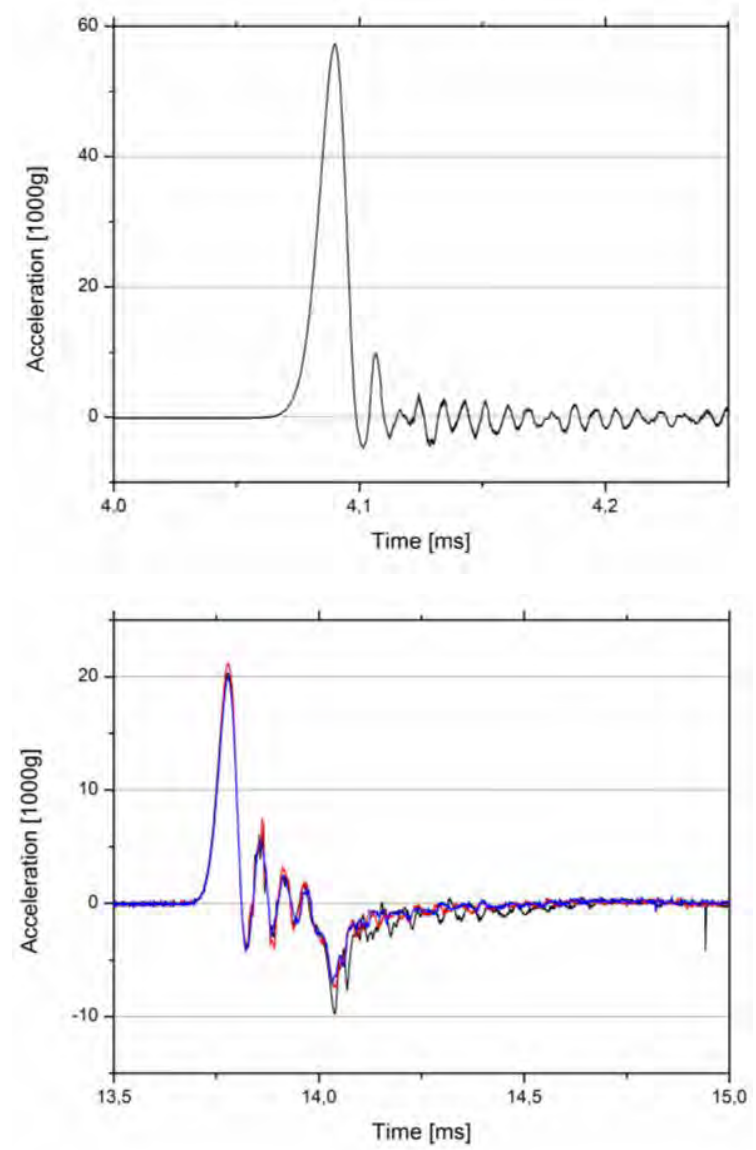

Fig. 6: Above: Sample experiment with a 60,000 g pulse generated on a Hopkinson bar. Below: Three consecutive measurements with medium amplitude, demonstrating good reproducibility. 
The performance of the new accelerometer was compared to two commercially available reference sensors. Table 1 summarizes the results with respect to the main design goals sensitivity and resonant frequency, the latter being proportional to bandwidth. The new design exhibits an increased sensitivity, while maintaining a very high resonant frequency, overcoming the traditional trade-off. Its figure of merit $\phi_{\text {design }}$ is almost one order of magnitude larger than for the reference designs. The high performance is due to the new displacement focused design approach, for which the geometrical constant forms the basis. Of course, $C$ only accounts for a part of the increase in the figure of merit. More details on this can be found in [6].

Tab. 1: Comparison of the new design and commercially available reference accelerometers.

\begin{tabular}{|l|l|l|l|}
\hline & $\begin{array}{l}\text { Sensitivity } \\
\text { in } \mu \mathrm{V} / \mathrm{V} / \mathrm{g}\end{array}$ & $\begin{array}{l}\text { Resonant } \\
\text { frequency }\end{array}$ & $\begin{array}{l}\phi_{\text {design }} \\
(\text { eq. }(1)) \\
\text { in 10 } 3 / \mathrm{m}\end{array}$ \\
\hline Ref. A & 0.21 & $164 \mathrm{kHz}$ & 23 \\
\hline Ref. B & 0.09 & $1.34 \mathrm{MHz}$ & 650 \\
\hline EMI & 0.50 & $1.5 \mathrm{MHz}$ & 4500 \\
\hline
\end{tabular}

\section{Generalization of Geometrical Constant}

The usefulness of a displacement focused design approach is clearly demonstrated by the high performance the new device exhibits. It is noteworthy that the geometrical constant, which forms the basis of the design strategy, has been known for decades but to the authors' knowledge never was applied in a sensor design. Since the geometrical constant is an inherent feature of every continuous springmass system, any sensor that uses mechanical oscillatory components can in principle benefit from it - so long as sensitivity and (mechanical) bandwidth play a role for its performance.

For all inertial sensors, like accelerometers or gyroscopes, no generalization needs to be done - the concept of the geometrical constant can simply be applied. However, for low bandwidth, high sensitivity sensors, it may not be possible to design systems that have an increased value of $C$. High sensitivity geometries usually demand a large mass on rather fragile bending elements, which always results in a constant close to ' 1 '. The strategy of generating a high- $C$ geometry and then scaling it, will likely run into dimension limitations, due to it becoming too large to be within the manufacturing limitations. For medium and high bandwidth sensors however, the concept should at least be considered. Not that the geometrical constant can also be determined for axial vibrations [11]. Regarding pressure sensors that usually feature flat, membrane like structures, high bandwidth and sensitivity are also desired [14]. An optimization with respect to $C$ can be carried out in much the same way as has been discussed here, since pressure acts uniformly on the system.

Resonant sensors, which need a driving force to work, might also benefit from a high geometrical constant. Unfortunately, underlying working principles are more complicated and eq. (4) cannot be applied any more. However, Sundararajan original formula accounts for general loading, not just inertial forces:

$$
C \approx \frac{1}{q_{0}} \frac{\int_{V} q \cdot \Delta x_{\text {norm }} d V}{\int_{V} \cdot \Delta x_{\text {norm }}^{2} d V}
$$

Here, $q$ is the generalized static loading per unit domain $V$ acting on the system and $q_{0}$ a constant to normalize the loading. Note that the formula can be applied, only if the static deflection curve is similar to the first vibration mode [10]. For inertial loading, this is automatically the case.

AFM probes are also subject to external forces and need to have good sensitivity and bandwidth [15] [16]. In this case, a point load is acting on the system.

\section{Conclusion and Summary}

The high performance of the new accelerometer demonstrates that the displacement focused design approach was successful. The basis of this strategy, the geometrical constant, allowed finding the new, promising spring-mass system geometry.

The geometrical constant seems almost like a forgotten parameter. Especially against the background that geometry optimization is almost exclusively conducted with numerical methods. Still, it provides insight for the design process on a medium abstraction level and allows the designer to quickly assess general spring-mass geometries.

The generality of this constant indicates that it may be successfully applied to other sensor types that have mechanical oscillatory parts. However, high-g accelerometers are probably the most important examples, since these have a specifically high requirement for combining high bandwidth and high sensitivity. 


\section{Acknowledgement}

The accelerometer development has been supported by the Federal Office of Bundeswehr Equipment, Information Technology and InService Support (BAAINBw). We thank our colleagues Ulrich Schaber, Andreas Drost, Robert Faul, Prof. Karlheinz Bock and Adrian Goldberg for conducting the silicon processing and package manufacturing, respectively.

\section{References}

[1] M.J. Forrestal, T.C. Togami, W.E. Baker and D.J. Frew. Performance evaluation of accelerometers used for penetration experiments. In SEM 2002, 2002.

[2] S. J. Cunningham, D. G. Mclntyre, J. S. Carper, P. D. Jaramillo and A. W. Tang. Microstructures designed for shock robustness. In SPIE Proceedings, pages 99-107, 1996.

[3] O. Millon, W. Riedel, K. Thoma, E. Fehling and M. Nöldgen. Fiber-reinforced ultra-high performance concrete under tensile loads. In 9th International Conference on the Mechanical Behaviour of Materials under Dynamic Loading, DYMAT, pages pg. 671677. EDP Science, 2009.

[4] S. Huang, X. Li, Z. Song, Y. Wang, H. Yang, $\mathrm{L}$. Che and J. Jiao. A high-performance micromachined piezoresistive accelerometer with axially stressed tiny beams. Journal of Micromechanics and Microengineering, 15(5):993, 2005. DOI: 10.1088/0960-1317/15/5/014

[5] N. Yazdi, F. Ayazi and K. Najafi. Micromachined inertial sensors. Proceedings of the IEEE, 86(8):1640 -1659, aug 1998. DOI: 10.1109/5.704269

[6] R. Kuells, S. Nau, M. Salk and K. Thoma. Novel piezoresistive high-g accelerometer geometry with very high sensitivitybandwidth product. Sensors and Actuators A: Physical, 182(0):41 - 48, DOI: 2012.10.1016/j.sna.2012.05.014

[7] W.M. Rubio, C.N. Silva and S. Nishiwaki. Piezoresistive sensor design using topology optimization. Structural and Multidisciplinary Optimization, 36:571-583, 2008. DOI: 10.1007/s00158-007-0191-6

[8] A.G. Krause, M. Winger, T.D. Blasius, Q. Lin and $O$. Painter. A high-resolution microchip optomechanical accelerometer. Nature Photonics, 6:768-772, 2012. DOI: 10.1038/nphoton.2012.245

[9] R. Jones. Approximate expressions for the fundamental frequency of vibration of several dynamic systems. Journal of Sound and Vibration, 44(4):475 - 478, 1976. DOI: 10.1016/0022-460X(76)90089-4
[10] C. Sundararajan. Relationship between the fundamental frequency and the static response of elastic systems. Journal of Sound and Vibration, 51(4):493 - 499, 1977. DOI: $10.1016 / S 0022-460 \times(77) 80047-$ 3

[11] C.W. Bert. Relationship between fundamental natural frequency and maximum static deflection for various linear vibratory systems. Journal of Sound and Vibration, 162(3):547 - 557, 1993. DOI: 10.1006/jsvi.1993.1139

[12] R. Kuells, S. Nau, M. Salk, K. Thoma, M. Ihle and A. Goldberg. Novel high-g accelerometer geometry requiring 90 degree contacting techniques. In IMAPS/ACerS 8th International Conference and Exhibition on Ceramic Interconnect and Ceramic Microsystems Technologies (CICMT 2012), Erfurt, 2012.

[13] H. Kolsky. Stress Waves in Solids. Dover Publications, 2003. unaltered reprint.

[14] P. Darlington. Gain bandwidth products for capacitive pressure transducers. Applied Acoustics, 41(4):365 - 375, 1994. Special Issue on Transducers. DOI: 10.1016/0003682X(94)90094-9

[15] K. Naeli and O. Brand. Coupling high force sensitivity and high stiffness in piezoresistive cantilevers with embedded sinanowires. In Sensors, 2007 IEEE, pages $1065-1068$, oct. 2007. DOI: 10.1109/ICSENS.2007.4388589

[16] T. Gotszalk, P. Grabiec and I.W. Rangelow. Calibration and examination of piezoresistive wheatstone bridge cantilevers for scanning probe microscopy. Ultramicroscopy, 97(1-4):385 - 389, 2003. Proceedings of the Fourth International Conference on Scanning Probe Microscopy, Sensors and Nanostructures. DOI: 10.1016/S0304-3991(03)00065-2 